\title{
Interaction between prenatal growth and high-risk genotypes in the development of type 2 diabetes
}

\author{
N. Pulizzi • V. Lyssenko • A. Jonsson • C. Osmond • \\ M. Laakso • E. Kajantie • D. J. Barker • L. C. Groop • \\ J. G. Eriksson
}

Received: 25 September 2008 / Accepted: 27 January 2009 / Published online: 19 February 2009

(C) Springer-Verlag 2009

\begin{abstract}
Aims/hypothesis Early environmental factors and genetic variants have been reported to be involved in the pathogenesis of type 2 diabetes. The aim of this study was to investigate whether there is an interaction between birthweight and common variants in the TCF7L2, HHEX, PPARG, KCNJ11, SLC30A8, IGF2BP2, CDKAL1, CDKN2A/2B and JAZF1 genes in the risk of developing type 2 diabetes.

Methods A total of 2,003 participants from the Helsinki Birth Cohort Study, 311 of whom were diagnosed with type 2 diabetes by an OGTT, were genotyped for the specified variants. Indices for insulin sensitivity and secretion were calculated.

Results Low birthweight was associated with type 2 diabetes $(p=0.008)$ and impaired insulin secretion $(p=0.04)$. Of the tested variants, the risk variant in HHEX showed a trend
\end{abstract}

Electronic supplementary material The online version of this article (doi:10.1007/s00125-009-1291-1) contains supplementary material, which is available to authorised users.

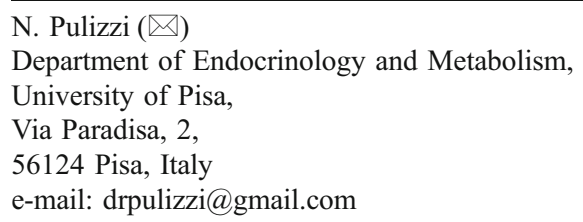

towards a low birthweight $(p=0.09)$ and the risk variant in the $C D K N 2 A / 2 B$ locus was associated with high birthweight $(p=0.01)$. The TCF7L2 risk allele was associated with increased risk of type 2 diabetes. Pooling across all nine genes, each risk allele increased the risk of type 2 diabetes by $11 \%$. Risk variants in the $H H E X, C D K N 2 A / 2 B$ and $J A Z F 1$ genes interacted with birthweight, so that the risk of type 2 diabetes was highest in those with lower birthweight $(p \leq 0.05)$. The interaction was also present in the pooled data.

Conclusions/interpretation Low birthweight might affect the strength of the association of some common variants (HHEX, CDKN2A/2B and JAZF1) with type 2 diabetes. These findings need to be replicated in independent cohorts.

Keywords Genetic variants · Interaction - Prenatal growth · Type 2 diabetes
E. Kajantie · J. G. Eriksson

National Public Health Institute, Helsinki, Finland

D. J. Barker

Oregon Health and Science University, Portland, OR, USA

J. G. Eriksson

Department of General Practice and Primary Healthcare, University of Helsinki,

Helsinki, Finland

J. G. Eriksson

Vasa Central Hospital,

Vasa, Finland 


$\begin{array}{ll}\text { Abbreviations } \\ \text { CIR } & \text { Corrected insulinogenic response } \\ \text { DI } & \text { Disposition index } \\ \text { HBCS } & \text { Helsinki Birth Cohort Study } \\ \text { PI/I ratio } & \text { Proinsulin/insulin ratio } \\ \text { S }_{\text {I }} & \text { Insulin sensitivity index } \\ \text { SNP } & \text { Single-nucleotide polymorphism }\end{array}$

\section{Introduction}

Previous studies have shown an association between small birth size and later type 2 diabetes [1]. Programming, the phenomenon whereby insults during early development produce physiological or morphological phenotypes that adversely affect later health, has been suggested to mediate this association [2]. Since insulin secretion regulates both fetal growth and glucose metabolism throughout life, it has been suggested that a common genetic denominator, possibly involving impaired insulin secretion, might predispose to both a small body size at birth and type 2 diabetes later in life.

The aim of this study was to assess whether the risk of type 2 diabetes might be influenced by an interaction between birthweight and common variants of genes recently identified to be associated with diabetes by genome-wide association studies, i.e. TCF7L2-rs7903146, HHEX-rs1111875, PPARG-rs1801282, KCNJ11-rs5219, SLC30A8-rs13266634, IGF2BP2-rs4402960, CDKAL1rs7754840, CDKN2A/2B-rs10811661 and JAZF1-rs864745 [3]. We decided to include only the $J A Z F 1$ gene from a list of six novel variants identified in a meta-analysis as only variants in $J A Z F 1$ predicted future type 2 diabetes in a large prospective cohort [4].

\section{Methods}

Helsinki Birth Cohort Study (HBCS) A total of 2,003 participants, 928 men and 1,075 women, aged 56-70 years, born as singletons from 1934 through to 1944, underwent an OGTT. Detailed information on the HBCS has been published previously [5]. Type 2 diabetes was defined according to WHO criteria (1999). Insulin sensitivity was estimated by the insulin sensitivity index $\left(\mathrm{S}_{\mathrm{I}}\right)$ [6]; beta cell function was assessed as an index of the corrected insulinogenic response (CIR) [7] and disposition index $\left(\mathrm{DI}=\mathrm{CIR} \times \mathrm{S}_{\mathrm{I}}\right)$ to adjust insulin secretion for $\mathrm{S}_{\mathrm{I}}$. Intact proinsulin concentration was measured at fasting and a proinsulin/insulin (PI/I) ratio was calculated. The reported investigations were carried out in accordance with the principles of the Declaration of Helsinki.
Genotyping Genotyping was performed using PCR-singlestrand conformation polymorphism for PPARG-rs1801282, competitive allele-specific PCR method (KASPar; KBioscience, Hoddesdon, UK) for SLC30A8-rs13266634 and a TaqMan allelic discrimination assay (Applied Biosystems, Foster City, CA, USA) for the other variants. We obtained a genotyping success rate of $>95 \%$ for all variants except rs4402960 (94\%). Risk alleles, defined upon previous association studies, are given in the Electronic supplementary material (ESM) Table 1. All genotypes were in HardyWeinberg equilibrium in non-diabetic participants. In diabetic participants IGF2BP2-rs4402960 deviated from Hardy-Weinberg equilibrium $(p=0.0068)$ : genotyping error is unlikely because after re-genotyping of 100 samples (5\%), the obtained concordance rate was $100 \%$.

Statistical analysis Data are presented as means $\pm \mathrm{SD}$ or median (interquartile range) for non-normally distributed variables. Only additive models were considered. Continuous variables were compared by analysis of covariance and group frequencies by a $\chi^{2}$ test. Multivariate linear regression analyses were used to test genotype-phenotype correlations, adjusted for age, sex and BMI. The effect of the genetic variant on birthweight was assessed by linear regression adjusting for sex, gestational age, parity, maternal age and BMI at delivery. Consequently for each variant only participants with complete data were included in the analysis. Type 2 diabetes risk was assessed by logistic regression analysis, adjusted for age, sex and BMI. The interaction between birthweight and genotype was assessed by creating a product variable. The combined genetic profile was calculated summing up the number of risk alleles of the nine variants (theoretically each person could carry a range of 0-18 risk alleles; ESM Fig. 1). Statistical analyses were performed by SPSS 15.0. A nominal $p$ value $<0.05$ was considered statistically significant.

\section{Results}

Characteristics of the study participants are shown in ESM Table 2. Of the 2,003 participants, $311(15.6 \%)$ had developed type 2 diabetes and 496 (24.8\%) impaired glucose tolerance. Type 2 diabetes was more frequent in men than in women $(19.5 \%$ vs $12.1 \%, p<0.01)$ and the risk increased with increasing BMI (OR per $1 \mathrm{~kg} / \mathrm{m}^{2}$ higher BMI: 1.16 [95\% CI 1.13, 1.19], $p<0.0001)$. A low birthweight, after adjustment for age, sex and BMI $(n=1,996)$, predicted future risk of type 2 diabetes (OR per $1 \mathrm{~kg}$ lower birthweight: 1.42 [95\% CI 1.09, 1.84], $p=0.008)$, as well as impaired insulin secretion $(p=0.04)$ and action $(p<0.0001)$ in adult non-diabetic participants. 
Table 1 Effect of genetic variants on birthweight

\begin{tabular}{|c|c|c|c|c|}
\hline Chr & Gene & SNP & Genotype $^{\mathrm{a}}(n)$ & $\begin{array}{l}\text { Birthweight } \\
\text { increase }(\mathrm{g}) \text { per } \\
\text { risk allele } \\
(95 \% \mathrm{CI})^{\mathrm{b}}\end{array}$ \\
\hline 10 & $T C F 7 L 2$ & rs7903146 & $\begin{array}{l}\text { CC; CT; TT } \\
\quad(1,028 ; 525 ; 67)\end{array}$ & $2(-33,36)$ \\
\hline 10 & HHEX & rs1111875 & $\begin{array}{l}\mathrm{AA} ; \mathrm{AG} ; \mathrm{GG} \\
\quad(352 ; 807 ; 452)\end{array}$ & $-24(-52,4)$ \\
\hline 3 & PPARG & rs 1801282 & $\begin{array}{l}\mathrm{GG} ; \mathrm{CG} ; \mathrm{CC} \\
\quad(59 ; 445 ; 1,156)\end{array}$ & $-14(-50,22)$ \\
\hline 11 & KCNJ11 & rs 5219 & $\begin{array}{l}\text { CC; CT; TT } \\
\quad(465 ; 744 ; 380)\end{array}$ & $-9(-36,18)$ \\
\hline 8 & SLC $30 A 8$ & rs 13266634 & $\begin{array}{l}\text { TT; CT; CC } \\
\quad(719 ; 809 ; 207)\end{array}$ & $-7(-36,22)$ \\
\hline 3 & $I G F 2 B P 2$ & rs4402960 & $\begin{array}{l}\mathrm{CC} ; \mathrm{AC} ; \mathrm{AA} \\
\quad(814 ; 592 ; 153)\end{array}$ & $23(-7,53)$ \\
\hline 6 & $C D K A L 1$ & rs7754840 & $\begin{array}{l}\text { GG; CG; CC } \\
\quad(687 ; 712 ; 179)\end{array}$ & $-9(-39,21)$ \\
\hline 9 & $C D K N 2 A / 2 B$ & rs10811661 & $\begin{array}{l}\text { GG; AG; AA } \\
\quad(34 ; 386 ; 1,158)\end{array}$ & $48(8,88)$ \\
\hline 7 & $J A Z F 1$ & rs864745 & $\begin{array}{l}\text { GG; AG; AA } \\
\quad(406 ; 829 ; 390)\end{array}$ & $4(-23,32)$ \\
\hline - & $\begin{array}{l}\text { Combined } \\
\text { risk }\end{array}$ & - & Per each risk allele & $-1(-12,10)$ \\
\hline
\end{tabular}

${ }^{\text {a }}$ Ordered by increasing risk of type 2 diabetes

${ }^{\mathrm{b}}$ Linear regression, adjusted for gestational age, sex, maternal age, maternal BMI and parity

$\mathrm{Chr}$, chromosome

Effect of genetic variants on risk of type 2 diabetes Only the risk T allele of the TCF7L2-rs7903146 was significantly associated with increased risk of type 2 diabetes (OR 1.47 [95\% CI 1.18, 1.82]). Other studied variants were not independently associated with increased risk of type 2 diabetes. Considering the combined genetic profile, highrisk genotypes were associated with increased risk of type 2 diabetes compared with low-risk genotypes, each risk allele increasing the odds by $11 \%(p=0.007)$ (ESM Table 1$)$.

Effect of genetic variants on insulin secretion and sensitivity The risk alleles (T and G) of TCF7L2 and HHEX were associated with impaired beta cell function (CIR: $p=0.008$ and $p=0.002$; DI: $p=0.01$ and $p=0.002$ ) compared with non-risk alleles ( $\mathrm{C}$ and $\mathrm{A})$. Additionally the risk $\mathrm{C}$ allele of $C D K A L 1$-rs 7754840 was associated with increased PI/I ratio ( $8 \%$ per risk allele, $p=0.0001$ ) compared with the $\mathrm{G}$ allele (ESM Table 3). None of the risk variants was associated with reduced insulin sensitivity (data not shown).

Effect of genetic variants on birthweight Only individuals with complete data on maternal and gestation features were included. The risk $\mathrm{G}$ allele of the $H H E X$-rs 1111875 showed a trend towards a lower birthweight $(-24[95 \% \mathrm{CI}-52,4]) \mathrm{g}$, $p=0.09$ ) compared with the A allele. In contrast, the risk A allele of $C D K N 2 A / 2 B$-rs 10811661 was associated with a $48 \mathrm{~g}(95 \%$ CI 8,87$)$ increase in birthweight $(p=0.01)$ compared with the $\mathrm{G}$ allele. Carriers of high-risk genotypes did not show a significant decrease in birthweight compared with carriers of low-risk genotypes (Table 1).

Interaction between genetic risk variants and birthweight in the prediction of type 2 diabetes risk Risk variants at the $H H E X, C D K N 2 A / 2 B$ and JAZF1 loci interacted with birthweight to predict future type 2 diabetes $(p=0.04,0.03$ and
Fig. 1 Effect size of the interaction between birthweight and genotype
TCF7L2

HHEX

IGF $2 B P 2$

CDKAL1

PPARG

KCNJ11

SLC $30 A 8$

$C D K N 2 A / 2 B$

$J A Z F 1$

Pooled
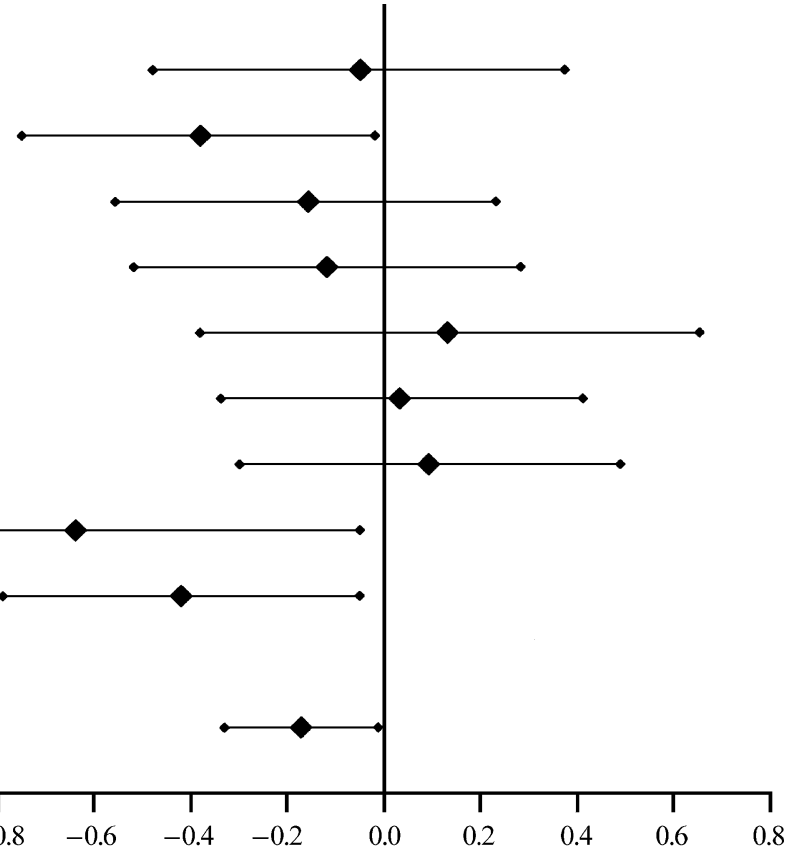

Interaction effect size 
0.02 , respectively): their effect on the risk of type 2 diabetes was more pronounced in individuals with a low birthweight (Fig. 1). Furthermore, individuals with the lowest birthweight and the most high-risk genotypes had the greatest risk of type 2 diabetes ( $p$ for interaction $=0.03$ ).

\section{Discussion}

The HBCS allows exploration of the effects of the intrauterine environment on glucose tolerance $>60$ years later. It provides an ideal scenario to assess whether the common variants in the susceptibility genes influence the established association between a smaller body size at birth and risk of type 2 diabetes. We focused upon the first variants reported to be associated with type 2 diabetes. Among variants reported in a recent meta-analysis, all of them with a small effect size, we genotyped only the variant that was previously shown to predict type 2 diabetes in a large prospective cohort $(J A Z F 1)$.

The key finding in this study was that a lower birthweight amplifies the risk conferred by the pooled variants in nine type 2 diabetes susceptibility genes. On the contrary, a higher birthweight seemed to counterbalance the risk conferred by the genetic background. Importantly, these risk estimates were corrected for and independently of adult BMI, which is known to interact with genotype in prediction of future type 2 diabetes [8].

The TCF7L2-rs7903146 single-nucleotide polymorphism (SNP) did not influence birthweight but was a strong independent predictor of type 2 diabetes. A recent metaanalysis indicated that there is an association between maternal risk genotype of this variant and birthweight, suggesting that the higher birthweight was determined through maternal alterations in glucose metabolism. The association between the fetal risk allele and birthweight was mainly due to the correlation with maternal genotype [9]. Unfortunately, in elderly participants from the HBCS we cannot trace the maternal genotype, nor do we have information on glucose metabolism during pregnancy.

In our study, only variants of $H H E X$ and $C D K N 2 A / 2 B$ seemed to influence birthweight, but in opposite directions. The reduced birthweight in carriers of the risk genotypes of $H H E X$, whose insulin secretion was reduced in adult life, points to the possibility that an early impairment of beta cell development and function would retard fetal growth in these participants, since insulin is a well-recognised anabolic factor during fetal life. This is extrapolated from insulin secretion in adult life, as we have no data on insulin secretion at earlier stages. Similarly, we were not able to test whether the higher birthweight observed in participants carrying the risk allele of $C D K N 2 A / 2 B$-rs10811661 might be mediated through maternal glycaemia. We could not replicate the association of this variant with an increased risk of future type 2 diabetes, but of the tested new type 2 diabetes loci, the risk conferred by the chromosome 9 locus seems to be the least reproducible [4].

We confirmed the association of common variants in TCF7L2 and HHEX with low insulin secretion and of $C D K A L 1$ with impaired proinsulin conversion [10].

A feature of the study is that there were only 311 cases of type 2 diabetes. This determines the precision with which it is possible to estimate the size of interaction effects, as shown by the width of the CIs in Fig. 1. However, this study has included more cases than have the majority of other such birth cohort studies.

In conclusion, common variants in susceptibility genes might interact with the intrauterine environment to increase the risk of future type 2 diabetes. These findings need to be replicated in independent cohorts.

Acknowledgements Work at the Lund University Diabetes Centre in Malmö was supported by a Linne grant from the Swedish Research Council as well as by grants from the European Community (ENGAGE), the Nordic Centre of Excellence in Disease Genetics and the Wallenberg Foundation. The HBCS has been supported by grants from the Academy of Finland, the British Heart Foundation, the Finnish Diabetes Research Foundation, the Finnish Foundation for Cardiovascular Research, the Finnish Foundation for Pediatric Research, the Finnish Medical Societies (Duodecim and Finska Läkaresällskapet), the Juho Vainio Foundation, the Päivikki and Sakari Sohlberg Foundation, the Signe and Ane Gyllenberg Foundation and the Yrjö Jahnsson Foundation.

Duality of interest The author declares that there is no duality of interest associated with this manuscript.

\section{References}

1. Barker DJ, Hales CN, Fall CH, Osmond C, Phipps K, Clark PM (1993) Type 2 (non-insulin-dependent) diabetes mellitus, hypertension and hyperlipidaemia (syndrome $\mathrm{X}$ ): relation to reduced fetal growth. Diabetologia 36:62-67

2. Hales CN, Barker DJ (1992) Type 2 (non-insulin-dependent) diabetes mellitus: the thrifty phenotype hypothesis. Diabetologia 35:595-601

3. Zeggini E, Scott LJ, Saxena R et al (2008) Meta-analysis of genome-wide association data and large-scale replication identifies additional susceptibility loci for type 2 diabetes. Nat Genet 40:638-645

4. Lyssenko V, Jonsson A, Almgren P et al (2008) Clinical risk factors, DNA variants, and the development of type 2 diabetes. $\mathrm{N}$ Engl J Med 359:2220-2232

5. Eriksson JG, Osmond C, Kajantie E, Forsen TJ, Barker DJ (2006) Patterns of growth among children who later develop type 2 diabetes or its risk factors. Diabetologia 49:2853-2858

6. Stumvoll M, van Haeften T, Fritsche A, Gerich J (2001) Oral glucose tolerance test indexes for insulin sensitivity and secretion 
based on various availabilities of sampling times. Diabetes Care 24:796-797

7. Hanson RL, Pratley RE, Bogardus C et al (2000) Evaluation of simple indices of insulin sensitivity and insulin secretion for use in epidemiologic studies. Am J Epidemiol 151:190-198

8. Lyssenko V, Almgren P, Anevski D et al (2005) Genetic prediction of future type 2 diabetes. PLoS Med 2:e345
9. Freathy RM, Weedon MN, Bennett A et al (2007) Type 2 diabetes TCF7L2 risk genotypes alter birth weight: a study of 24,053 individuals. Am J Hum Genet 80:1150-1161

10. Kirchhoff K, Machicao F, Haupt A et al (2008) Polymorphisms in the TCF7L2, CDKAL1 and SLC30A8 genes are associated with impaired proinsulin conversion. Diabetologia $51: 597-601$ 